\title{
Successful use of Infliximab in macrophage activation syndrome with severe CNS affection
} A Sellmer*1,2,3, B Stausbøl-Grøn ${ }^{1}$ and T Herlin ${ }^{1}$

\author{
Address: ${ }^{1}$ Department of Pediatrics, Randers Hospital, Randers, Denmark, ${ }^{2}$ Magnetic Resonance Centre, Aarhus University Hospital Skejby, \\ Aarhus, Denmark and ${ }^{3}$ Department of Pediatrics, Aarhus University Hospital Skejby, Aarhus, Denmark \\ * Corresponding author
}

\author{
from I5th Paediatric Rheumatology European Society (PreS) Congress \\ London, UK. 14-17 September 2008 \\ Published: 15 September 2008 \\ Pediatric Rheumatology 2008, 6(Suppl I):P200 doi:I0.II86/I546-0096-6-SI-P200
}

This abstract is available from: http://www.ped-rheum.com/content/6/SI/P200

(C) 2008 Sellmer et al; licensee BioMed Central Ltd.

Macrophage activation syndrome (MAS) is a secondary form of haemophagocytic lymphohistiocytosis (HLH) seen in childhood in the context of rheumatic disease. MAS is a serious complication to systemic onset juvenile idiopathic arthritis (SoJIA) with significant morbidity and mortality.

We present a 12 year old boy admitted to hospital with a 2 week history of fever, malaise, muscle tenderness, and skin rash. Initially an infection was suspected based on symptoms and an exceptional high CRP (3717 nmol/l), however despite antibiotics and a rapidly decreasing CRP the clinical status deteriorated. The patient became somnolent and rigid in all four extremities having weak deep tendons reflexes. Laboratory studies showed an elevated CRP $(2158 \mathrm{nmol} / \mathrm{l})$, relative pancytopenia, coagulopathy, high serum ferritin $(1254 \mu \mathrm{mol} / \mathrm{l})$, and increasing liver transaminases. MRI of the cerebrum showed progressive lesional changes on T2-weighted FLAIR sequence in the cortex and basal ganglia. Bone marrow examination was normal, and did not show hemophagocytosis.

MAS based on SoJIA was suspected and treatment with cyclosporine A (4 mg/kg/day) and high dose methylprednisolone (12 mg/kg/day for 3 days) was initiated. The patient did not respond to initial treatment. Anti-TNF- $\alpha$ monoclonal antibody Infliximab $(5 \mathrm{mg} / \mathrm{kg}$ ) was added to the treatment and repeated after 2 and 6 weeks. The patient rapidly improved clinically and biochemically and went into full recovery after four months.
MAS complicated with severe CNS involvement is associated with an extremely high mortality rate. Our case supports the addition of infliximab when standard treatment fails. 